\title{
GRN Gene
}

National Cancer Institute

\section{Source}

National Cancer Institute. GRN Gene. NCI Thesaurus. Code C101401.

This gene is involved in cytokine-like activity. 